\title{
Fundamental aspects of SXES in the Quantification of Minerals and Materials
}

\author{
Nicholas Wilson ${ }^{1}$, Colin MacRae ${ }^{2}$ and Aaron Torpy ${ }^{1}$ \\ ${ }^{1}$ CSIRO Mineral Resources, Clayton, Australia, ${ }^{2}$ CSIRO Mineral Resources, Clayton, Victoria, Australia
}

Electron probe microanalysers (EPMAs) are fitted with wavelength dispersive spectrometers (WDS) and quite often energy dispersive spectrometers (EDS) for the elemental quantification of samples. In recent years, soft $\mathrm{x}$-ray emission spectrometers (SXES) comprising of a grating and CCD camera have been fitted to EPMAs primarily for the identification of light elements and to measure peak shape and position changes due to bonding. With the SXES's parallel x-ray detection, and high energy resolution (Fig. 1a), they also offer the possibility of quantification. [1-3]

In addition to parallel detection, the SXES also offers other advantages over the WDS. In the case of SXES the gratings are typically fixed, so they do not suffer mechanical reproducibility problems that can occur in WDS. Also, using a CCD camera, the SXES detector does not suffer from count loss due to dead time (Fig. 1c), although this is normally easily solved on WDS detectors with a correction factor (Fig. 1b).

However, there are several drawbacks using the SXES detector for quantification. Depending upon the grating being used, higher order reflections of lines can be a problem. While the higher order lines, with their increased energy resolution, can be useful in spectroscopy, they can cause interferences with trying to perform quantification, and unlike in WDS detectors, they cannot be removed by energy filtering. The SXES detector also suffers from a lower count at a particular energy, however this is partially offset by its parallel detection of a range of energies.

More problematic are the new sources of noise not present in WDS. Since there is no window between the CCD and the chamber, stray electrons can cause noise peaks in the spectrum, although these stray electrons can be minimised using apertures and deflection magnets. Cosmic rays can also strike the CCD generating false counts. CCDs also suffer from spurious thermally generated electrons, called "dark current", but this can be minimized by cooling the CCD and is not a large problem in this application. The largest problem is that of CCD read noise.

In a CCD, photons are not counted individually, they build up charge in the CCD which is read out periodically as a voltage, and the read noise comes from errors in reading this voltage. Read noise can be minimised by repeated measurements, so there is a benefit in taking more shorter measurements for an analysis rather than fewer longer measurements. Figure 2 plots histograms of fifty analyses of the $\mathrm{Al} \mathrm{K} \alpha$ line measured with different acquisition timing. In Fig 2 (a) the CCD is sampled every $0.5 \mathrm{~s}$ and it can be seen there is a reduction in the error of the analysis by extending the total measurement time from 20s to 40s. In Fig. 2 (b) the analyses are done for a total of 40s but in the second set the CCD is only sampled every $5 \mathrm{~s}$. The total number of photons captured in each set in (b) was the same, but the CCD was read ten times less when the sample rate was $5 \mathrm{~s}$, leading to a larger read noise component.

\section{References}

1. Takahashi H. Characteristic Features and Applications of a Newly Developed Wavelength Dispersive Soft X-ray Emission Spectrometer for Electron Probe X-ray Microanalyzers and Scanning Electron Microscopes. JEOL News. 2014.

2. Takahashi H, McSwiggen P, Nelson C. A Unique Wavelength-dispersive Soft X-ray Emission spectrometer for electron probe X-ray microanalysers. Microscopy and Analysis. 2014.

3. Moy A, Fournelle J, von der Handt A. Quantitative Measurement of Iron-Silicides by EPMA Using the Fe L $\alpha$ and L $\beta$ X-ray Lines: A New Twist to an Old Approach. Microsc Microanal. 2019;25(3):664-74. 


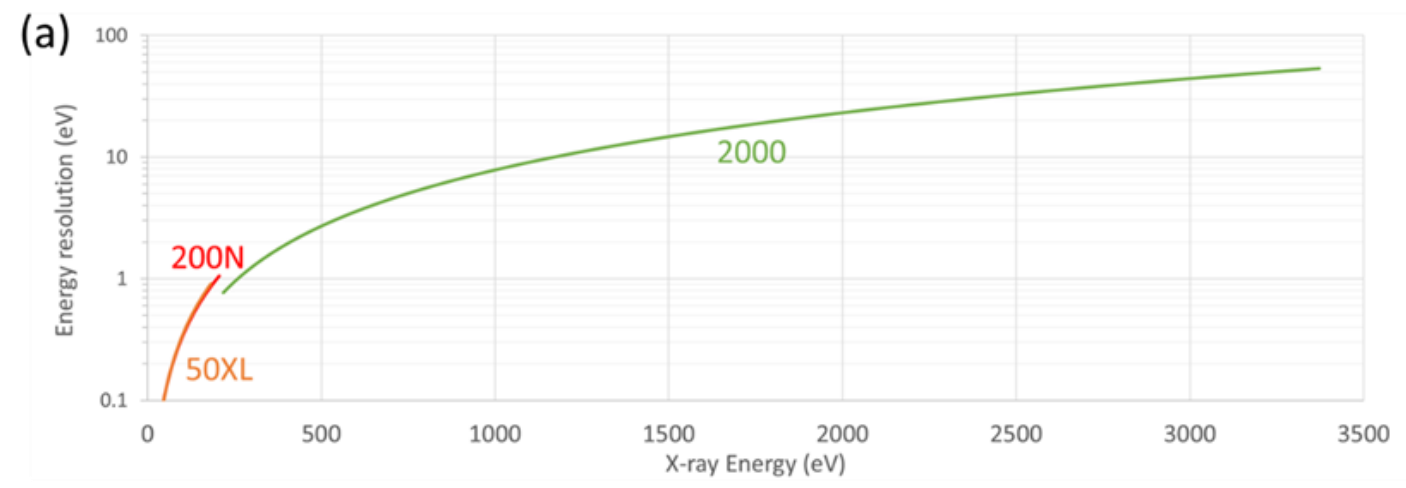

(b)

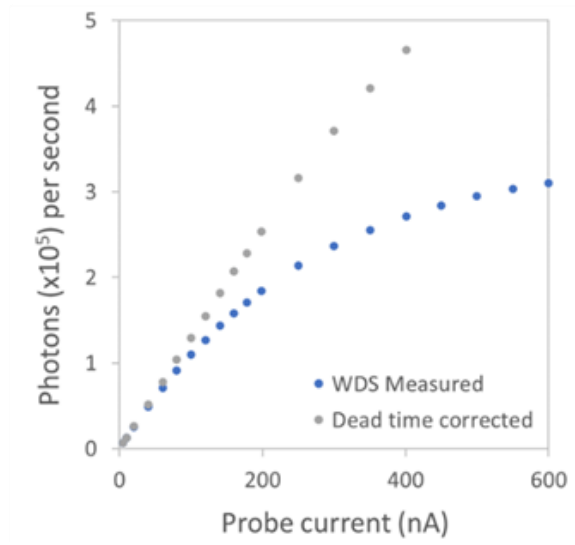

(c)

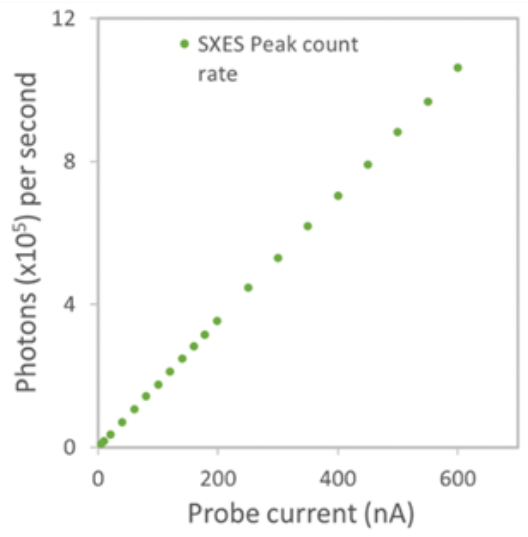

Figure 1. (a) Energy resolution of first order lines versus $x$-ray energy for the $50 \mathrm{XL}, 200 \mathrm{~N}$ and 2000 soft $\mathrm{x}$-ray gratings. (b) Peak carbon K $\alpha$ count rate versus beam current using WDS LDE2 crystal, (c) integral 3rd order carbon $\mathrm{K} \alpha$ peak rate using $200 \mathrm{~N}$ grating.
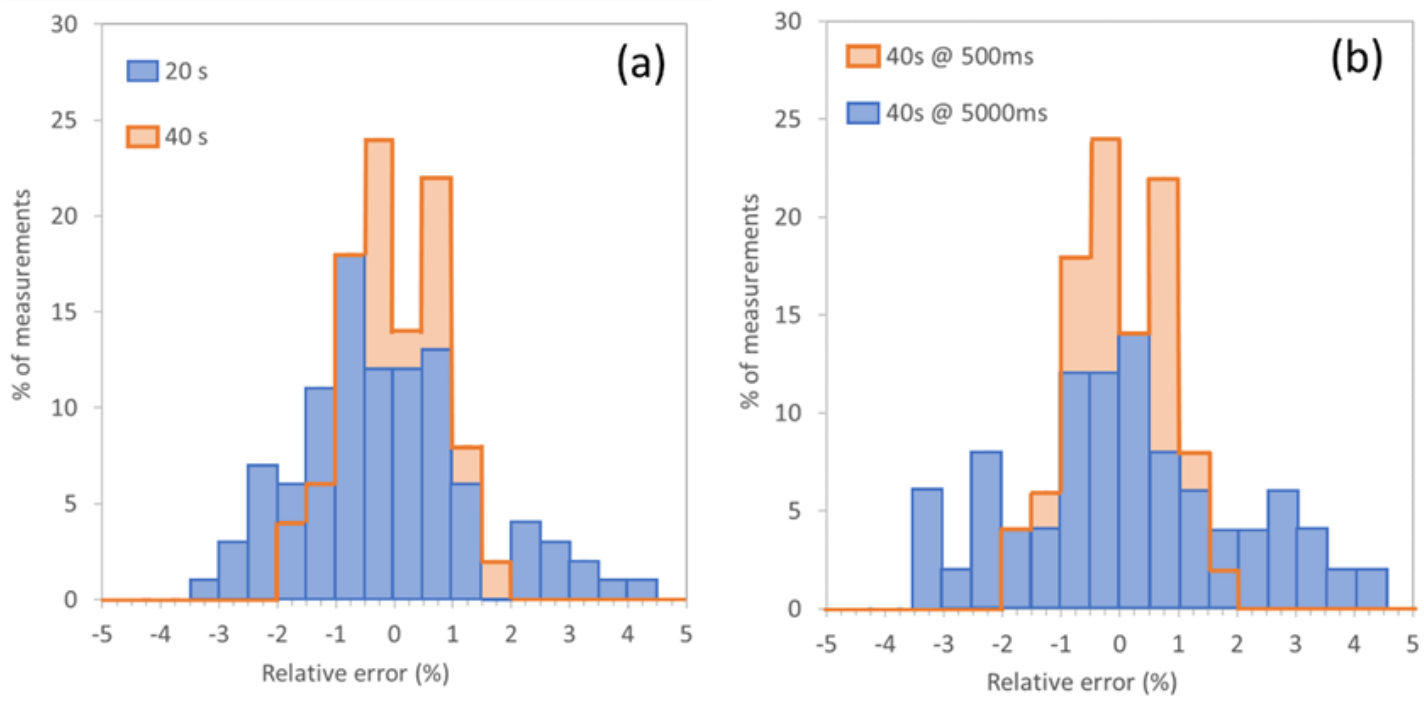

Figure 2. Absolute error histograms. (a) comparison of $20 \mathrm{~s}$ and $40 \mathrm{~s}$ total acquisition at $0.5 \mathrm{~s}$ sampling rate. (b) comparison of $40 \mathrm{~s}$ total acquisition with $0.5 \mathrm{~s}$ and $5 \mathrm{~s}$ sampling. Al metal $\mathrm{K} \alpha$ line measured on metal at $15 \mathrm{kV}$ using the 2000 grating. 